The Cardiology 14 (1-4): 1-6, 2019

ISSN: $1811-8194$

(C) Medwell Journals, 2019

\title{
Examining the Gallstone Existence in Patients with Coronary Artery Stenosis Compared to Non-Coronary Artery Stenosis
}

\author{
${ }^{1}$ Mehrnoosh Boroomandpoor and ${ }^{2}$ Nika Hajatpour \\ ${ }^{1}$ Department of Cardiovascular Medicine, Tehran Medical Sciences, \\ ${ }^{2}$ Young Researchers and Elite Club, Tehran Medical Sciences, \\ Islamic Azad University, Tehran, Iran
}

\begin{abstract}
Coronary heart disease is one of the common diseases and causes of many deaths in the world. Hence, recognizing the risk factors involved in development of this disease and its prevention as well as fast and timely diagnosis prevent many deaths in the world. An important practice is preventing cardiovascular diseases by recognizing the factors associated with them and reducing the burden of disease by identifying diseases associated with coronary artery disease. In this regard, biliary diseases are associated with cardiovascular diseases. Thus, given the importance of this issue, this research was conducted to evaluate the presence of gallstones in patients with coronary artery stenosis compared to non-coronary artery stenosis in $\mathrm{Bu}$ Ali Hospital during 2015-2016. In this observational and descriptive-analytical and cross-sectional study, 83 patients undergoing angiography in Bu Ali Hospital during 2015-2016 were examined. The patients with coronary artery stenosis were compared with the patients without coronary artery stenosis in terms of presence or absence of stones and gallbladder inflammation through sonography. The results revealed that 70 patients $(84.3 \%)$ had coronary artery stenosis. Gallstone in ultrasound was observed in 23 patients $(27.7 \%)$. Gallstone in ultrasound was seen in 20 patients $(28.6 \%)$ in coronary artery stenosis group and 3 patients $(23.1 \%)$ in non-coronary artery stenosis which showed no significant difference $(p>0.05)$. Based on the results, it is concluded that the presence of gallstone in patients with coronary artery stenosis is not significantly different from that in patients without coronary artery stenosis.
\end{abstract}

Key words: Gallstone, gallbladder, coronary artery stenosis, angiography, ultrasound, significant

\section{INTRODUCTION}

Coronary Artery Disease (CAD) is considered as the leading cause of death in most communities such as United States, so that, more than one million new and recurrent cardiovascular events occur annually in the United States and it is expected that the prevalence and complications of this disease to increase.

Developments in the treatment of this disease have increased the survival of patients after the first cardiovascular event but patients with CAD are at higher risk for subsequent cardiovascular events (Bainey and Jugdutt, 2009; Weinstein et al., 1987; Delahaye et al., 2003). In patients with CAD, the most important predictors of survival include left ventricular function and the extent to which the myocardium is at risk. Left ventricular dysfunction represents undesirable prognosis. This dysfunction might be due to congestive heart failure in the examination or in the form of an ejection fraction drop in echocardiography or ventriculography.

Moreover as the severity of the dysfunction is higher, the prognosis would be worse (Koch et al., 2003). Myocardial ischemia is a condition in which the supply of oxygen to the myocardium is insufficient to meet its metabolic needs. This imbalance would lead to CAD, so that, in the presence of a constant stenosis in the coronary artery, the distal vessel may be expanded (or close to the maximum) at rest state as far as possible. During periods of increased demand, the artery will have little ability for dilation (reduction in reserve of coronary artery dilation) which will lead to a mismatch between supply and demand and consequently is chemia. In addition, angina is also formed in cases of coronary stenosis which limits the flow of blood and is caused in response to an increase in oxygen demand in the myocardium following physical or psychological pressure. A treatment approach to angina involves modifying risk factors, changing lifestyle, drug therapy and vascular regeneration. Given the symptoms and effects of CAD, control of hypertension, diabetes and hyperlipidemia and smoking cessation are important in control of the progression of the disease in people with coronary atherosclerosis. Moreover, the effects of organic nitrates in controlling the angina have been known for more than one century and these drugs are still among the most common anti-angina treatment prescribed in patients with chronic angina. The effects of nitrates are applied by relaxing smooth vascular muscles. 
The dilatation of arterioles also decreases the general artery resistance. The effect of these drugs on the venous system is deeper. Veins dilatation leads to blood accumulation in the veins, reduced venous return and reduced preload. Epidemiological studies have also identified several factors which increase the likelihood of atherosclerosis formation in a given individual. High age, male gender and family history of early atherosclerosis are considered as unchangeable risk factors. The rate of coronary heart disease prevalence increases with increasing the age. Other risk factors are largely modifiable and change in these factors might reduce the risk of atherosclerosis. Diabetes mellitus also increases the risk of CAD and CAD-related mortality, so that, in the absence of diabetes, insulin resistance and hyperinsulinemia are associated with increased CAD which is probably due to changes in lipid metabolism and increased platelet aggregation.

Cigarette smoke also has a negative impact on the pattern of fats, clotting factors and motor function and it is associated with a two to four times of increase in the risk of CAD.

Given the causes of incidence and symptoms, CAD treatment is nowadays based on three methods: medical treatment which is the primary treatment of these patients, Percutaneous Coronary Intervention (PCI) which is less invasive compared to the coronary artery bypass graft surgery and its cost-effective and associated with fewer complications, Coronary Artery Bypass Graft surgery (CABG). Given what was stated above, recognizing the risk factors involved in development of this disease and preventing them as well as their early diagnosis are crucial issues in this area which reduces the mortality of many people. Important method for preventing cardiovascular diseases is recognizing the factors associated with them and reducing the burden of the disease by identifying diseases associated with coronary artery disease. Biliary diseases such as gallstones are among the diseases which are associated with cardiovascular disease.

The main substances of gallstone include cholesterol, biliary pigments and calcium. Other elements include iron, phosphorus, carbonates, proteins, carbohydrates, mucus and cellular debris.

Gallstones are formed as result of sedimentation of solids in a solution. Moreover, the increased degradation in red blood cells after replacing the valves leads to the formation of gallstone. Gallstones are formed in the absence of infections or inflammation but infection is not a major cause of the development of gallstones. In addition, stasis which means temporary bile flow passage to intestine and its staying in the gallbladder, plays a major role in the development of gallstones.

Temporary bile stasis may be due to dysfunctions or mechanical blockage in the meeting point of choledochal, duodenum or gallbladder. In addition, patients with asymptomatic gallstones should not be treated and dyspepsia, burping and bloating are not considered as a specific symptom. On the other hand, several studies have been conducted with regard to coronary and biliary diseases and the relationship between these two diseases has been investigated from several aspects.

In a retrospective study conducted by Lee et al. (2013) to evaluate the increased risk of biliary events in patients with gallstone without gallbladder symptom in the presence of coronary artery disease, results showed that the risk of biliary events associated with gallbladder was significantly high in CAD patients. Also, in patients with asymptomatic gallstones, coronary artery disease can increase the risk of biliary events associated with stone, especially, cholecystitis. Finally, it was recommended that patients with CAD to be monitored accurately, even if they are asymptomatic (Lee et al., 2013).

In a prospective study conducted by Lv et al. (2015) to evaluate the association between gallstones and ischemic heart disease, they finally, realized that presence of stones is associated with an increased risk of ischemic heart disease after statistical analysis and examination of patients in terms of presence of stones in patients with ischemic disease and without it. In another prospective cohort study conducted by Zheng et al. (2016) to evaluate the gallstone and risk of coronary heart disease, it was revealed that a history of gallstone disease increases the risk of coronary heart disease by $23 \%$. This research was carried out to evaluate and compare the presence of gallstone stone in patients with coronary artery stenosis, compared with patients without coronary artery stenosis in Bu Ali Hospital in years 2015 and 2016.

\section{MATERIALS AND METHODS}

In this observational study conducted as a descriptive-analytical and cross-sectional way, 83 patients who were suspected for coronary artery stenosis underwent angiography. Angiography was performed in $\mathrm{Bu}$ Ali hospital during 2015 and 2016. They were included into study using randomized and convenient sampling method. The patients with coronary stenosis on angiography were compared with patients without coronary artery stenosis in terms of presence or absence of gallbladder inflammation and gallstone by sonography. The inclusion criteria of patients were coronary angiography or gallbladder sonography and the exclusion criteria were lack of angiography or lack of gallbladder sonography. Patients underwent sonography $24 \mathrm{~h}$ after angiography by a specialist radiology team and sonography device Aloka SSD 3500 SX with a convex probing at a frequency of 3-5 MHz. They were examined in terms of the presence or absence of gallbladder inflammation and gallstone. It should be noted that, 
patients should not eat food at least $8 \mathrm{~h}$ and should not fatty food for 12-18 h. Patients should not take any drug affecting the biliary system (thiazide diuretics, ceftriaxone, cyclosporine, anticholinergics, narcotics, dapsone, OCP, blood cholesterol-lowering drugs and opioids). Then, patients were divided into coronary artery stenosis and non-coronary artery stenosis groups and the results of sonography were compared in terms of the presence or absence of gallstones in these two groups.

In addition, the association between age, gender, family history, heart disease and history of hypertension, history of diabetes, smoking and BMI and the presence or absence of gallstones was examined in this study. Finally, after collecting the required data from all subjects, they were analyzed using SPSS 13 Software. The frequency of gallstones in patients with coronary artery stenosis was compared with that of patients without coronary artery disease by Chi-square and Fisher tests and the significance level was considered to be 0.05 , so that, $\mathrm{p}<0.05$ was considered significant and $\mathrm{p}>0.05$ was considered non-significant.

\section{RESULTS AND DISCUSSION}

A total of 83 patients underwent angiography in $\mathrm{Bu}$ Ali Hospital that 70 (84.3\%) suffered from coronary artery stenosis and 13 of them $(15.7 \%)$ had no coronary artery stenosis (Fig. 1).

In this study, 83 subjects underwent angiography after $24 \mathrm{~h}$ under gallbladder sonography. Out of the 83 subjects, 23 subjects $(27.7 \%)$ had gallstones in sonography and 60 subjects $(72.3 \%)$ reported no gallstone in sonography (Fig. 2).

Based on the investigations, 70 patients had coronary artery stenosis which 20 of them (28.6\%) had gallstone and $50(71.4 \%)$ of them had no gallstone. In 13 patients without coronary artery stenosis, 3 cases $(23.1 \%)$ had gallstone and 10 cases $(76.9 \%)$ had no gallstones which did not show a significant difference $(\mathrm{p}>0.05)$ (Fig. 3).

In an investigation on 83 patients underwent angiography, one case had age below 40 years who had no coronary artery stenosis but had gallstone. In this study, 42 of patients aged 40-60 years which 10 of them had coronary artery stenosis along with gallstone. The 27 cases had coronary artery stenosis without gallstones. The 1 case of gallstone was reported without coronary artery disease and 4 cases had neither coronary artery stenosis nor gallstone ultrasonography. There were 40 cases of patients over the age of 60 who had 1 case of coronary artery stenosis and gallbladder stones. The 23 cases of coronary artery stenosis were reported without stones.

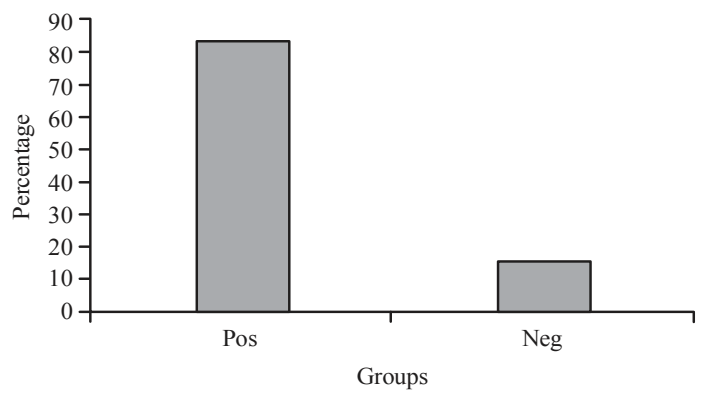

Fig. 1: Frequency distribution of coronary artery stenosis in patients (pos: positive, neg: negative)

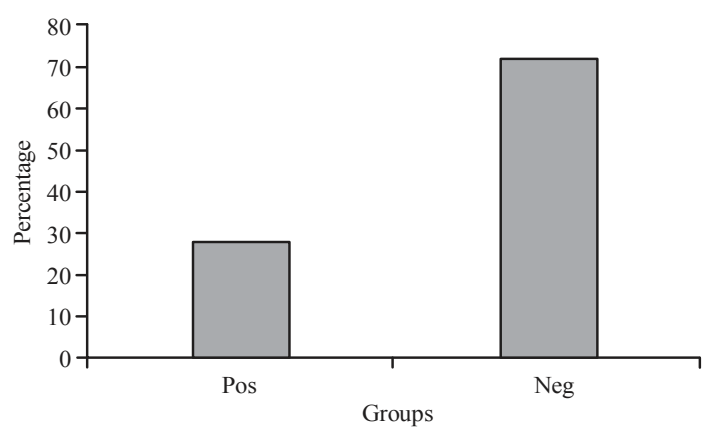

Fig. 2: Distribution of frequency of gallstones in sonography of patients (pos: positive, neg: negative)

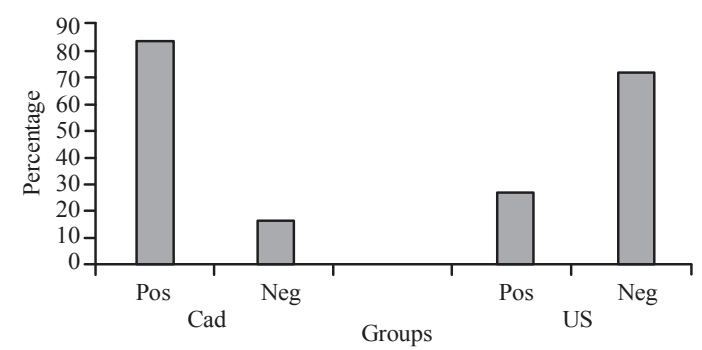

Fig. 3: Distribution of frequency of gallstones in patients with and without coronary artery stenosis (pos: positive, neg: negative, cad: coronary artery stenosis, us: gallstone)

One case of gallstone without coronary artery disease was reported and 6 cases had neither coronary artery stenosis nor gallstone. The frequency distribution of gallstones in patients with and without coronary stenosis was not significantly different from based on the age (p>0.05) (Fig. 4).

Out of 83 patients underwent angiography, 52 cases were male and 31 were female. Out of 52 male patients, 14 had gallstones and coronary artery disease and 32 cases had coronary artery disease without gallstone. Only 2 cases had gallstone and 4 did not have any of them. Out of 31 female subjects, 6 cases had gallstone and 


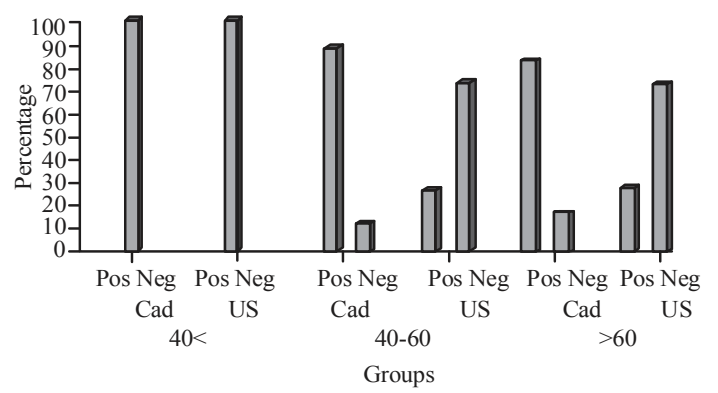

Fig. 4: Distribution of gallstones in patients with and without coronary stenosis based on age (pos: positive, neg: negative, cad: coronary artery, us: gallstone)

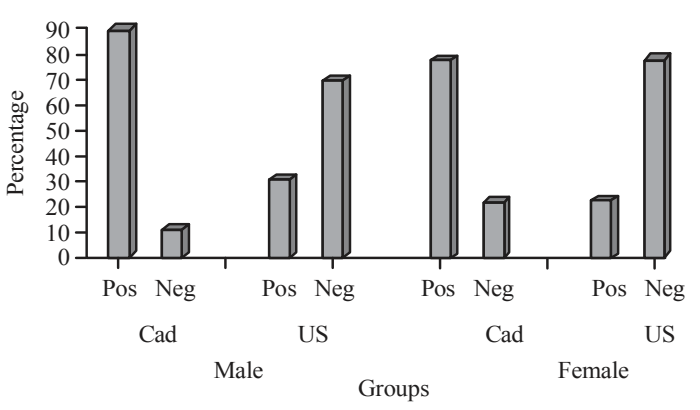

Fig. 5: Frequency distribution of gallstones in patients with and without coronary stenosis based on gender (pos: positive, neg: negative, cad: coronary stenosis, us: gallstone)

coronary artery disease and 18 cases had coronary artery disease and only 1 case had gallstone disease and 6 cases did not have any of them. The frequency distribution of gallstones in patients with and without coronary stenosis was not significantly different based on gender $(\mathrm{p}>0.05)$ (Fig. 5).

Ten patients had BMI between 20 and 24.99 which 1 had both gallstone and coronary artery disease, 5 had only coronary artery disease and 1 patient had only gallstone and 3 had none of them. In addition, 66 patients had BMI between 25 and 30 which 17 of them had both gallstone and coronary artery disease, 40 had only coronary artery disease, 2 had only stones and 7 had none of them. Seven patients had BMI over 30 which 2 of them had both diseases and 5 patients had coronary artery disease. Frequency distribution of gallstone in patients with without coronary stenosis was not significantly different based on BMI (p>0.05) (Fig. 6).

In total, 2 patients were diabetic and 54 patients were non-diabetic. Among the diabetics, 8 patients had both of the diseases, 16 had only coronary artery disease, 1 had only gallstone and 4 had none of them. Out of 54 non-diabetic patients, 12 cases had both of the diseases,

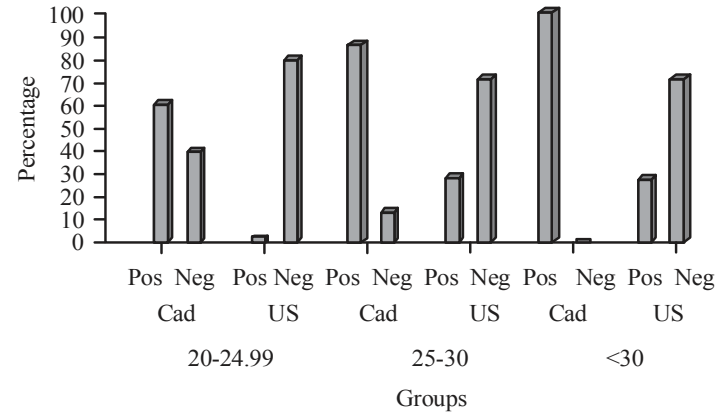

Fig. 6: Frequency distribution of gallstones in patients with and without coronary stenosis based on BMI (pos: positive, neg: negative, cad: coronary artery stenosis, us: gallstone)

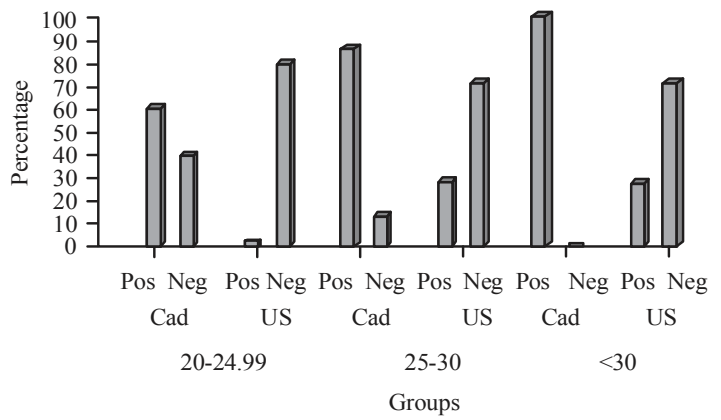

Fig. 7: Frequency distribution of gallstones in patients with and without coronary stenosis based on diabetes (pos: positive, neg: negative, cad: coronary artery stenosis, us: gallstone)

34 cases had only coronary artery disease, 2 cases had only gallstone and 6 had none of them. Frequency distribution of gallstone shows not significant difference between patients with coronary stenosis and patients without coronary stenosis based on diabetes $(p>0.05)$ (Fig. 7).

In this study, 50 patients had HTN which 11 of them had both of the diseases and 31 of them had only coronary artery disease and 8 patients had none of the diseases. In addition, 33 patients did not have HTN as an underlying disease which 9 of them had both of the diseases and 19 of them had only coronary artery disease, 3 of them had only gallstone and 2 cases had none of them. The frequency distribution of gallstones in patients with and without coronary stenosis was showed no significant difference based on hypertension ( $p>0.05$ ) (Fig. 8).

In this research, 17 subjects were non-smokers and 66 subjects were smokers. In non-smoker group, 5 cases had both of the diseases, 11 cases had only coronary artery disease and 1 patient did not have any of the diseases. In the smoker group, 15 patients had both of the diseases, 39 patients had only coronary artery disease, 3 patients had only gallstone and 9 cases did not have any 
The Cardiol., 14 (1-4): 1-6, 2019

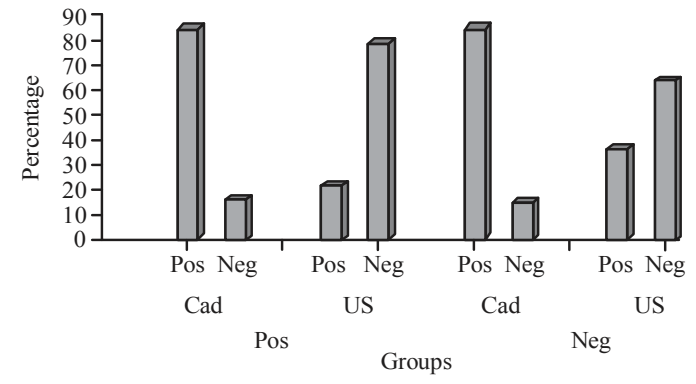

Fig. 8: Distribution of gallstones in patients with and without coronary stenosis based on hypertension (pos: positive, neg: negative, cad: coronary artery stenosis, us: gallstone)

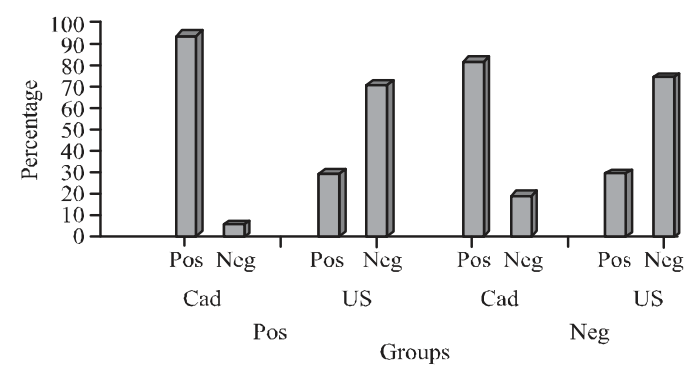

Fig. 9: Frequency distribution of gallstones in patients with and without coronary stenosis based on smoking (pos: positive, neg: negative, cad: coronary artery stenosis, us: gallstone)

of them. The frequency distribution of gallstones in patients with and without coronary stenosis shows no significant difference based on smoking $(\mathrm{p}>0.05)$ (Fig. 9).

In this research, 21 patients had a positive family history of heart disease which 5 of them had both of diseases, 12 of them had only coronary artery disease and 4 of them had none of the diseases. In addition, 62 patients did not have positive family history of heart disease which 15 of them had both of the diseases, 38 of them had only coronary artery disease, 3 of them had only gallstone and 6 of them had none of them. The frequency distribution of gallstones in patients with and without coronary stenosis shows no significant difference based on family history ( $\mathrm{p}>0.05)$ (Fig. 10).

An important practice in this regard is preventing cardiovascular diseases is recognizing the factors associated with them and reducing the burden of disease by identifying diseases associated with coronary artery disease. In this regard, biliary diseases are associated with cardiovascular diseases (Lilly and Braunwald, 2012). Thus, given the importance of this issue, this research was conducted to evaluate the presence of gallstones in patients with coronary artery stenosis compared to non-coronary artery stenosis in Bu Ali Hospital in years

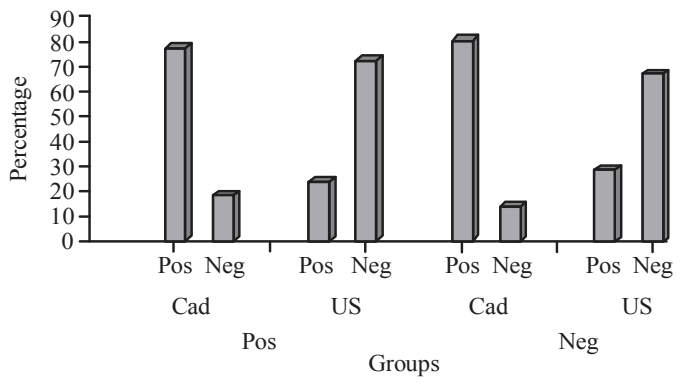

Fig. 10: Frequency distribution of gallstones in patients with and without coronary stenosis based on family history (pos: positive, neg: negative, cad: coronary artery stenosis, us: gallstone)

2015 and 2016. The results of this research revealed that 70 patients $(84.3 \%)$ had coronary artery stenosis and gallstone was seen in sonography of 23 patients $(27.7 \%)$. Gallstone was seen in sonography of $20(28.6 \%)$ patients with coronary artery stenosis and in $3(23.1 \%)$ patients without coronary artery stenosis which showed no statistically significant difference. In a retrospective cohort research conducted by Lee et al. (2013) in South Korea in 2013, 126 patients with coronary artery disease were compared with 252 patients without coronary artery disease which $33.5 \%$ in the first group and $27.5 \%$ in the second group had gallstone which showed a statistically significant difference with p-value of 0.017 . Thus, this result is not consistent with that of our research which can be due to the cross-sectional nature of our research (Lee et al., 2013). In a cohort study conducted by Olaiya et al. (2013) in Taiwan in 2013, 6981 patients had gallstone patients and 27924 patients had no gallstone which 935 patients $(13.4 \%)$ in the first group and 2758 patients $(9.8 \%)$ in the second group had coronary artery disease, showing significant difference (Lilly and Braunwald, 2012). This result is not in line with result of our research (Olaiya et al., 2013). In an analytical study conducted by Chavez-Tapia et al. (2012) in Mexico in 2012, a total of 798 patients reported that there was no significant difference in the frequency of coronary artery disease in patients with and without gallstone which is in line with results of our research. In a cross-sectional study conducted by Mendez-Sanchez et al. (2008) in Mexico in 2005, 119 patients with gallstone were compared with 354 patients without gallstone and it was found that $16 \%$ in the gallstone group and $4.5 \%$ in the non-gallstone group had coronary artery disease which significant difference was seen with $p<0.001$. This result is not consistent with the results of our research which is due higher power of their research (Mendez-Sanchez et al., 2008). In a review study conducted by Dickerman et al. (1991) in the United States in 1991, it was reported that there was a significant relationship between the presence of gallstones and coronary artery stenosis while our study did not reveal such result. 
In a cross-sectional study conducted by Jiang et al. (2013) in the UK, the results of the study showed that gallbladder stones are highly correlated with coronary artery disease diagnosed with angiography which is not in line with result of our study.

\section{CONCLUSION}

In general, based on the results of this study research and comparing them with other studies, it is concluded that there the presence of gallstone in patients with coronary artery stenosis compared to that in patients without coronary artery stenosis is not significantly different. It is finally, recommended that further studies with a larger sample size to be carried out to confirm the results of this study. Finally, as stated above, prevention is crucial in cardiovascular disease in order to reduce the burden of the disease. For prevention, the risk factors associated with it should be considered which gallbladder diseases are very important in this regard. In addition, taking preventive actions is very important.

\section{REFERENCES}

Bainey, K.R. and B.I. Jugdutt, 2009. Increased burden of coronary artery disease in South-Asians living in North America: Need for an aggressive management algorithm. Atherosclerosis, 204: 1-10.

Chavez-Tapia, N.C., I. Mac Kinney-Novelo, S.E. Sifuentes-Renteria, M. Torres-Zavala and G. Castro-Gastelum et al., 2012. Association between cholecystectomy for gallstone disease and risk factors for cardiovascular disease. Ann. Hepatol., 11: 85-89.

Delahaye, F., O. Roth and G. de Gevigney, 2003. Epidemiology of acute coronary syndrome. Rev. Prat, 53: 607-610.

Dickerman, J.L., 1991. Gallbladder disease and coronary artery disease: Is there a link? J. Am. Osteopathic Assoc., 91: 359-361.
Jiang, Z.Y., X. Sheng, C.Y. Xu, W.W. Li and X.X. Chang et al., 2013. Gallbladder gallstone disease is associated with newly diagnosed coronary artery atherosclerotic disease: A cross-sectional study. PLoS One, 8: 1-7.

Koch, C.G., Y.S. Weng, S.X. Zhou, J.S. Savino and J.P. Mathew et al., 2003. Prevalence of risk factors and not gender per se, determines short-and long-term survival after coronary artery bypass surgery. J. Cardiothoracic Vasc. Anesthesia, 17: 585-593.

Lee, Y.S., S.E. Jang, B.S. Lee, S.J. Lee and M.G. Lee $e t$ al., 2013. Presence of coronary artery disease increases the risk of Biliary events in patients with asymptomatic gallstones. J. Gastroenterol. Hepatol., 28: 1578-1583.

Lilly, L.S. and E. Braunwald, 2012. Braunwalds Heart Disease: A Textbook of Cardiovascular Medicine. 9th Edn.,/Vol. 2, Elsevier, New York, USA., ISBN:9781455711475, Pages: 312.

Lv, J., L. Qi, C. Yu, Y. Guo and Z. Bian et al., 2015. Gallstone disease and the risk of ischemic heart disease. Arteriosclerosis Thrombosis Vasc. Bio., 35: 2232-2237.

Mendez-Sanchez, N., D. Zamora-Valdes, J.A. Flores-Rangel, J.A. Perez-Sosa and F. Vasquez-Fernandez et al., 2008. Gallstones are associated with carotid atherosclerosis. Liver Intl., 28: 402-406.

Olaiya, M.T., H.Y. Chiou, J.S. Jeng, L.M. Lien and F.I. Hsieh, 2013. Significantly increased risk of cardiovascular disease among patients with gallstone disease: A population-based cohort study. PLoS One, 8: $1-8$.

Weinstein, M.C., P.G. Coxson, L.W. Williams, T.M. Pass and W.B. Stason et al., 1987. Forecasting coronary heart disease incidence, mortality and cost: The coronary heart disease policy model. Am. J. Pub. Health, 77: 1417-1426.

Zheng, Y., M. Xu, Y. Li, A. Hruby and E.B. Rimm et al., 2016. Gallstones and risk of coronary heart disease: Prospective analysis of 270,000 men and women from 3 US cohorts and meta-analysis. Arterioscler. Thromb. Vasc. Bio., 36: 1997-2003. 